\title{
CARACTERIZACIÓN MORFOLÓGICA Y ESTRUCTURAL DE POLVOS DE CENIZAS VOLANTES
}

\author{
(Morphological and structural characterization of fly ash powders)
}

"Facultad de Ciencias Básicas. Departamento de Física. Grupo GITEC. Universidad Francisco de Paula Santander, Cúcuta, Colombia. gpenaro@ufps.edu.co "Facultad de Ingenierías. Plan de Estudios de Ingeniería de Minas. Universidad Francisco de Paula Santander, Cúcuta, Colombia.

\section{(Recibido: 5 de Agosto de 2014 y aceptado 1 de Octubre de 2014)}

\begin{abstract}
Resumen:
Se reporta la caracterización morfológica y estructural de polvos de cenizas volantes obtenidos de la combustión de carbón, suministrados por la Central Termoeléctrica Termotasajero S.A. El estudio morfológico consistió en el análisis superficial mediante el uso de microscopia electrónica de barrido (MEB). La composición química elemental fue hallada usando los espectros de dispersión de energías de rayos X (EDS-MEB), mientras que la caracterización estructural se llevó a cabo por difracción de rayos X (DRX). Para la distribución del tamaño de partícula se usó el software Image Tool V. 3.0. Los resultados obtenidos muestran que los polvos de cenizas volantes están formados por partículas mayoritariamente esféricas (cenosferas y plerosferas), así como de partículas de inquemados adheridas a las partículas de ceniza, con tamaño de partícula promedio del orden de micrómetros. El estudio estructural, para el cual se utilizó DRX, reporta las fases cristalinas mayoritarias de la mullita, cuarzo y hematita. Nuestros resultados permitirán evaluar los procesos de combustión del carbón de la Central Termoeléctrica de Termotasajero, así como otros usos o aplicaciones a dichas cenizas.
\end{abstract}

Palabras clave: cenizas volantes, morfología, estructura, MEB, EDS, DRX

\section{Abstract:}

Morphological and structural characterization of fly ash powder's reports are obtained from coal combustion supplied by the thermal - electrical plant Termotasajero S.A. The morphological study consisted in the superficial analysis, using Scanning electron microscopy (SEM). The basic chemical composition was found using $\mathrm{X}$ ray energy dispersion spectrums (EDX - SEM) whereas structural characterization was developed by X-ray diffraction (XRD). The software Image tool V. 3.0. was used for the particle size distribution. The results obtained show that the fly ash powders are composed mostly by spherical particles as well as unburned particles adhered to the particles, with average particle size of order micrometers. The structural study, using XRD, reports the main crystalline phases of mullite, quartz and hematite. Our results will allow us to evaluate the Thermal - electrical Plant Termotasajero's coal combustion processes, as well as another uses and applications of those ashes.

\section{INTRODUCCIÓN}

En la producción de energía termoeléctrica, la combustión del carbón genera grandes cantidades de residuos sólidos, dentro de los que se encuentran las cenizas volantes, las cuales, en la actualidad, debido a su granulometría muy fina $(1$ a $100 \mu \mathrm{m})$ y a su actividad puzolánica, son usadas en la industria del cemento (Davidovits, 1994; Hanehara, Tomosawa, Kobayakawa \& Hwang, 2001; Bouzoubaa, Zhang \& Malhotra, 2001); también por su alta superficie específica se han usado como adsorbentes en la eliminación de colorantes (Wang, Boyjoo, Choueib \& Zhu, 2005) y en la remoción de sales y ácidos de boro (Öztürk \& Kavak, 2005) presentes en soluciones acuosas, desechadas por las industrias de tintas, de cueros y cosméticas. En la industria cerámica se han usado cenizas volantes clase $\mathrm{F}$, para elaborar ladrillos refractarios y pisos, cementos geopoliméricos de alta temperatura y en la producción de zeolitas por procesos hidrotermales (Rajamannan, Kalyana, Viruthagiri \& Shanmugam, 2013; Škvára, Jílek \& Kopecký, 2005; Ruiz-Román et al., 2000).
Keywords: flay ash, morphology, structure, SEM, EDS, XRD. 
ZAF a los espectros de dispersión de energías de rayos $\mathrm{X}$ (EDS-MEB) y la estructura, aplicando difracción de rayos X, a polvos de ceniza volante de la Central Termoeléctrica de Termotasajero S.A.

\section{MATERIALES Y MÉTODOS}

Los polvos de cenizas volantes fueron suministrados por la central térmica de Termotasajero S.A. Para el estudio de la morfología de dichos polvos, se utilizó el microscopio electrónico de barrido (MEB), marca FEI Quanta modelo $650 \mathrm{FEG}$, y el sistema de recubrimiento de muestras por pulverización catódica Quorum 150R-ES, el cual fue utilizado para metalizar las muestras con oro, y así evitar que se cargaran durante el barrido electrónico. Para la distribución granulométrica se usó el software libre Image Tool versión 3.0.

El microanálisis por elemento químico se realizó por corrección ZAF a los espectros de dispersión de energías de rayos X (EDS), para lo cual se usó la sonda EDAX serie SDD-Apollo X, adaptada al microscopio electrónico. Dicho análisis se efectuó en forma puntual tanto para las partículas de cenizas volantes como para los inquemados presentes en la muestra.

La estructura fue estudiada usando el método de polvos a través del difractómetro de rayos $\mathrm{X}, \mathrm{X}$-PertPro MPD Panalytical. Las especificaciones técnicas usadas en la toma de los patrones de difracción fueron: voltaje de aceleración $45 \mathrm{kV}$, intensidad de corriente de $40 \mathrm{~mA}$, longitud de onda $(\lambda=1.540598$ ? $)$ correspondiente a la línea $\mathrm{k}_{\alpha}$ del cobre $(\mathrm{Cu}-$ $\left.\mathrm{K}_{a}\right)$, tiempo de paso de 8 segundos, tamaño de paso de $0.02^{\circ}$ en modo continuo de $2 \mathrm{O}=10^{\circ}$ a $2 \mathrm{O}=80^{\circ}$ grados, en una configuración geométrica Bragg-Brentano. La preparación de la muestra para el análisis estructural consistió en tamizado de los polvos de cenizas volantes a través de tamiz ASTM 325, y el pasante se depositó en el portamuestras, el cual se niveló usando un vidrio portaobjetos.

\section{RESULTADOS Y DISCUSIÓN}

En la Figura 1, se presenta una microfotografía de la muestra de cenizas volantes a 500X. En esta se detalla la morfología superficial de la muestra, compuesta de partículas esféricas, las cuales corresponden a la ceniza volante, y de partículas irregulares aglomeradas correspondientes a inquemados. Lo anterior puede observarse con mayor claridad en la Figura 2, que corresponde a la microfotografía a 1000X de la muestra, donde se aprecian las partículas esféricas de ceniza volante (A), así como de partículas de menor tamaño adheridas a las de ceniza volante, que corresponden a los inquemados (B), los cuales dependen de la velocidad y temperatura de la combustión, así como del grado de pulverización y del tipo de carbón (Alonso, 1990).

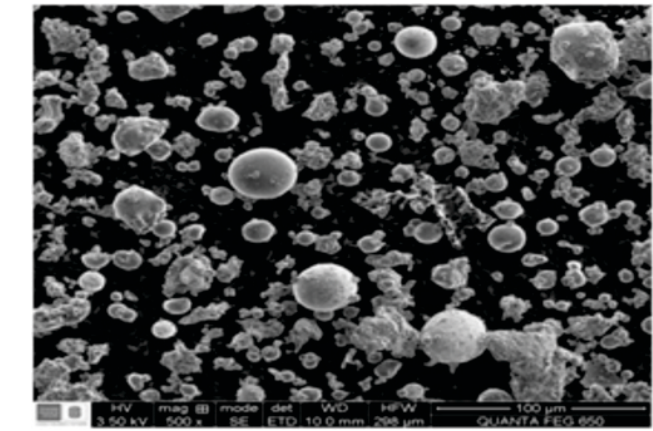

Figura 1. Microfotografía usando MEB a 500X de polvos de ceniza.

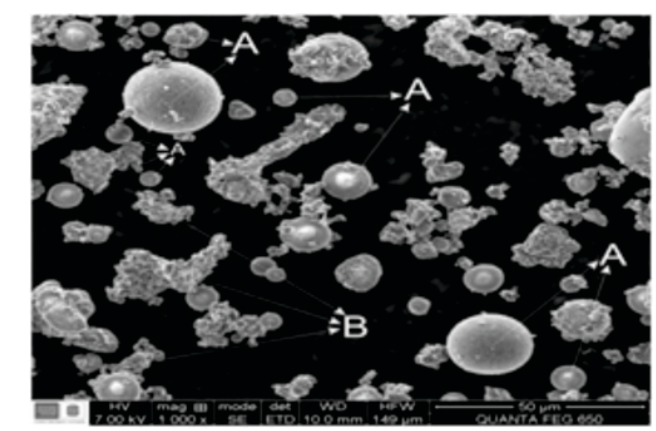

Figura 2. Microfotografía usando MEB a 1000X de polvos de ceniza.

Las cenosferas son pequeñas partículas esféricas macizas o huecas, mientras que las plerosferas son cenosferas huecas que contienen otras partículas esféricas de menor tamaño en su interior (Cabrera \& Cusens, 1982). En la Figura 3 se presenta una microfotografía a $5000 \mathrm{X}$, en la que se aprecian los diámetros de tres cenosferas de ceniza volante, con dimensiones de 6,61, 11,59 y 13,81 $\mu$ m respectivamente. La imagen de una plerosfera se exhibe en la Figura 4, donde se observa una esfera hueca que contiene partículas más pequeñas en su interior.

En general, la morfología de la muestra de ceniza volante de Termotasajero está conformada por partículas suaves al tacto, de color grisáceo, principalmente de forma esférica, que pueden ser vítreas, sólidas, huecas, lisas o esponjosas. También se aprecian partículas redondeadas con superficies picoteadas e irregulares, unidas a partículas de ceniza volante de menor tamaño (partículas aglomeradas de carbón o inquemados), y de cenosferas y plerosferas.

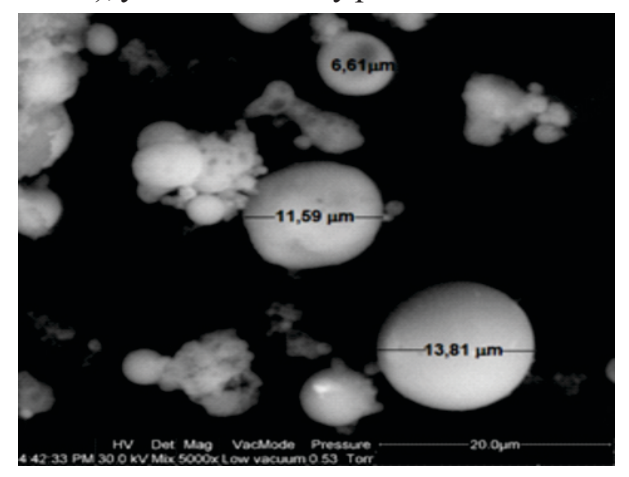

Figura 3. Microfotografía de partículas de ceniza volante (cenosferas), usando MEB a 5000X 


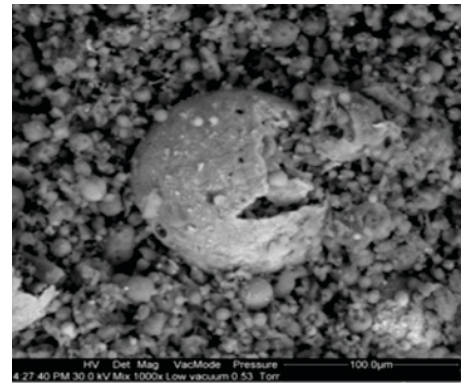

Figura 4. Microfotografía de una plerosfera de ceniza volante, usando MEB.

La distribución granulométrica de las partículas de ceniza volante se llevó a cabo usando el software libre Image Tool ${ }^{\circledR}$ versión 3.0, que permitió realizar la estadística de los diámetros promedios a las partículas de la muestra en estudio, para lo cual se utilizó la imagen tomada con el MEB, presentada en la Figura 1. La distribución del tamaño de partícula se hizo midiendo los diámetros de cada partícula horizontal, vertical y diagonalmente. Se encontró que el tamaño promedio de las partículas presentes en la Figura 1 es de $13.41 \mu \mathrm{m}$, con una desviación estándar de 0,54 , valor mínimo de 4,69 $\mu \mathrm{m}$, y un valor máximo de 33,40 $\mu \mathrm{m}$. Lo anterior permite inferir que la muestra presenta una distribución granulométrica heterogénea de partículas de cenizas volantes, con tamaños promedios de partícula que oscilan entre 4,69 y 33,40 $\mu \mathrm{m}$. Dicho resultado es confirmado con lo reportado en la literatura, donde se afirma que las cenizas volantes tienen granulometría con tamaño de partícula inferiores a $250 \mu \mathrm{m}$, y que del 60 al $90 \%$ son inferiores a $75 \mu \mathrm{m}$ (Cabrera \& Cusens, 1982).

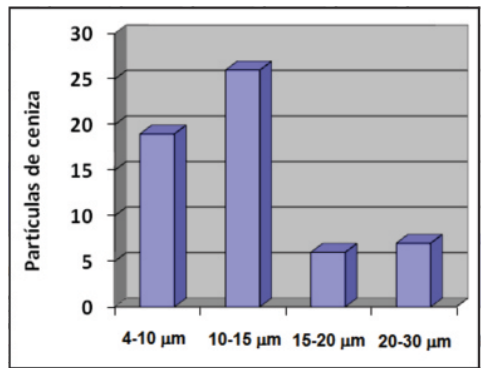

Figura 5. Diagrama de barras de la distribución granulometría de los polvos de cenizas volantes de Termotasajero S.A.

El diagrama de barras de la distribución granulométrica se presenta en la Figura 5. En esta se agruparon los tamaños de partículas en los rangos de 4 a $10 \mu \mathrm{m}, 10$ a $15 \mu \mathrm{m}, 15$ a $20 \mu \mathrm{m}$ y 20 a $30 \mu \mathrm{m}$. Se observa que la mayor cantidad de partículas está en el rango de 10 a $15 \mu \mathrm{m}$, seguido de las partículas en el rango de 4 a $10 \mu \mathrm{m}$, y en menor concentración partículas con rango entre 15 a $20 \mu \mathrm{m}$ y 20 a $30 \mu \mathrm{m}$, lo cual confirma lo reportado para el tamaño promedio que fue de $13,41 \mu \mathrm{m}$.

La distribución granulométrica de una muestra de ceniza volante permite conocer la reactividad química, así como la demanda de agua, cuando se agrega a una mezcla de cemento.
La granulometría de la ceniza volante se ve fuertemente influenciada por el proceso térmico usado en la combustión del carbón, y el grado de pulverización del carbón.

El microanálisis químico usando EDS, se realizó en forma puntual tanto para las partículas esféricas de ceniza volante, como para los inquemados presentes en la muestra. En la Figura 6 se presenta la ubicación puntual del cañón de electrones sobre una esfera de ceniza volante, para realizar el microanálisis químico. El espectro de dispersión de energías para esta partícula se presenta en la Figura 7, el cual se cuantifica en la Tabla 1. De ésta se observa que el elemento químico mayoritario de la partícula de ceniza volante es el $\mathrm{Fe}$, seguido del $\mathrm{Si}$ y $\mathrm{Al}$, y en menor concentración se encuentran el $\mathrm{Ti}, \mathrm{Ca}, \mathrm{K}, \mathrm{Cu}, \mathrm{Mg}, \mathrm{y}$ Na. Elementos que al mezclarse con el oxígeno formaron los diversos óxidos presentes en la muestra.

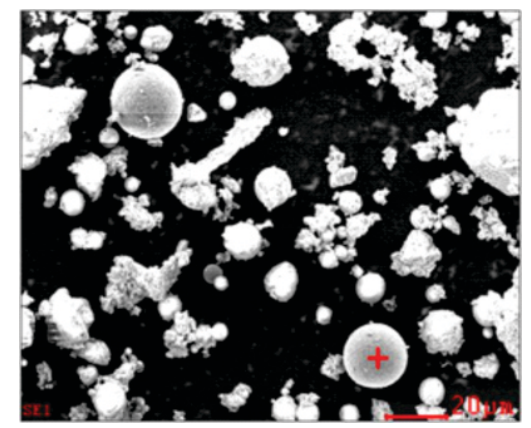

Figura 6. Imagen con la posición $(+)$ sobre una partícula de ceniza volante utilizada para cuantificar la composición química usando EDS

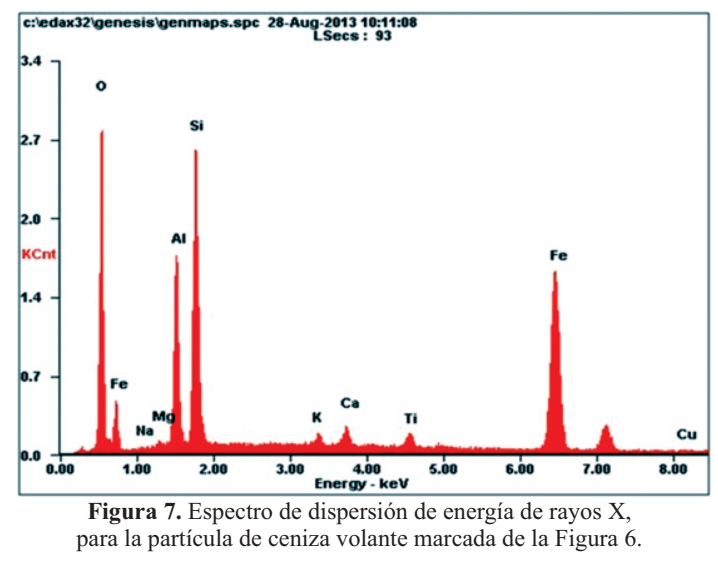

Tabla 1. Porcentaje en peso (Wt \%) y atómico (At \%), de la composición química para la partícula de ceniza volante marcada en la Figura 6.

\begin{tabular}{||c|c|c|}
\hline Element & W\% & At\% \\
\hline $\mathbf{O}$ & 26,83 & 47,07 \\
\hline $\mathbf{N a}$ & 00,19 & 00,23 \\
\hline $\mathbf{M g}$ & 00,42 & 00,48 \\
\hline $\mathbf{A l}$ & 11,30 & 11,76 \\
\hline $\mathbf{S i}$ & 18,37 & 18,36 \\
\hline $\boldsymbol{K}$ & 00,83 & 00,60 \\
\hline $\mathrm{Ca}$ & 01,40 & 00,98 \\
\hline $\mathrm{I} i$ & 01,45 & 00,85 \\
\hline $\mathrm{Fe}$ & 38,56 & 19,38 \\
\hline $\mathrm{Cu}$ & 00,66 & 00,29 \\
\hline Matrix & Correction & ZAF \\
\hline
\end{tabular}
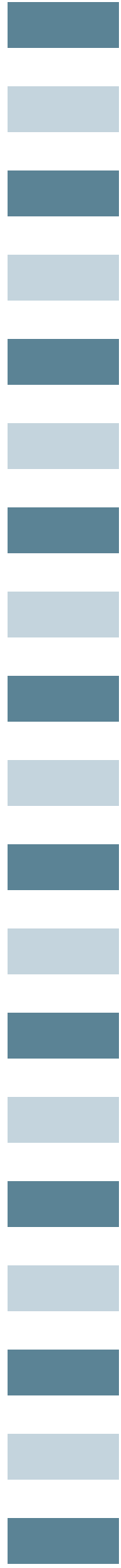
En forma similar se efectuó el microanálisis puntual sobre las partículas de inquemados presentes en la muestra de ceniza volante (ver marca $(+)$ en la Figura 8 ), el cual se cuantificó en la Tabla 2, del espectro de dispersión de energías presente en la Figura 9.

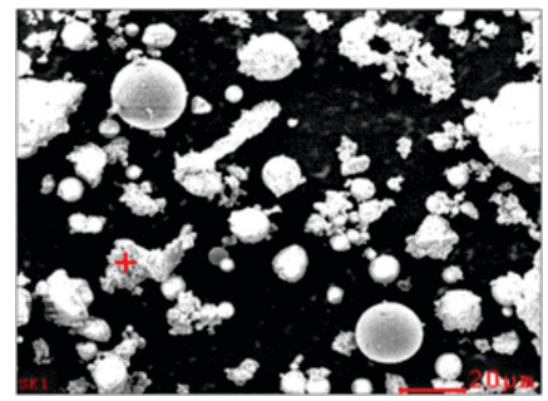

Figura 8. Imagen con la posición $(+)$ sobre partículas de inquemados utilizada para cuantificar la composición química usando EDS.

Al comparar el espectro de la Figura 7 con el presentado en la Figura 9, se aprecia la presencia del pico correspondiente al carbono (C), lo cual ratifica que dichas partículas corresponden a inquemados del carbón. De los resultados reportados en la Tabla 2 , se aprecia que el elemento químico mayoritario es el carbono, lo cual ratifica lo afirmado en el análisis de la morfología de la muestra, donde se confirma una vez más la presencia de carbón (inquemados) en la muestra de ceniza volante objeto de estudio. También se aprecia la presencia en menor concentración de $\mathrm{Si}, \mathrm{Al}, \mathrm{Fe}$ y pequeñas concentraciones de $\mathrm{K}, \mathrm{Ti}, \mathrm{Ca}$ y $\mathrm{Mg}$.

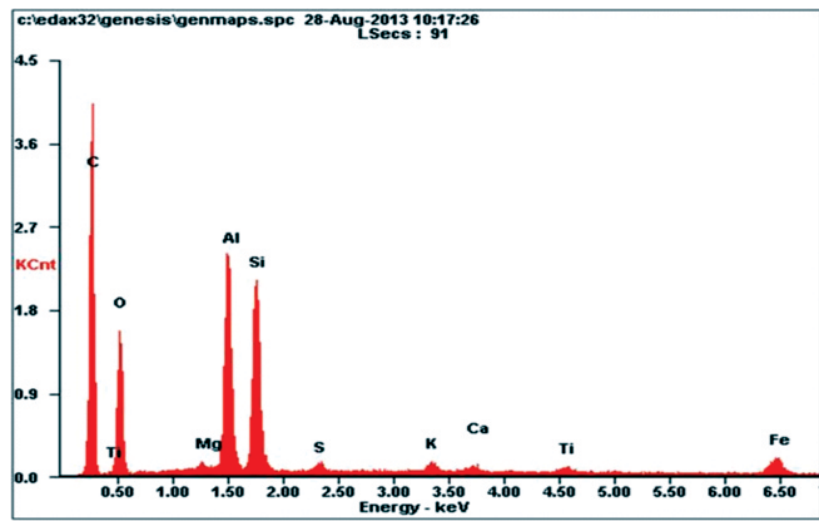

Figura 9. Espectro de dispersión de energía de rayos X, para las partículas de inquemados marcada de la Figura 8.

Tabla 2. Porcentaje en peso (Wt \%) y atómico (At \%), de la composición química para las partículas de carbón (inquemados) marcada en la Figura 8.

\begin{tabular}{||c|c|c||}
\hline Element & $W \tau \%$ & At\% \\
\hline$C$ & 53.31 & 67.02 \\
\hline$O$ & 22.12 & 20.88 \\
\hline$M g$ & 00.27 & 00.17 \\
\hline$A l$ & 09.05 & 05.07 \\
\hline$S i$ & 09.29 & 05.00 \\
\hline$S$ & 00.47 & 00.22 \\
\hline$K$ & 00.70 & 00.27 \\
\hline$C a$ & 00.51 & 00.19 \\
\hline Ii & 00.69 & 00.22 \\
\hline Fe & 03.59 & 00.97 \\
\hline Matrix & Correction & ZAF \\
\hline
\end{tabular}

Del análisis cuantitativo por elemento químico realizado usando espectroscopia de dispersión de energías de rayos X (EDS), se concluye que las cenizas de Termotasajero son silicoaluminosas con propiedades puzolánicas clase $\mathrm{F}$ (según norma ASTM C618), por tener porcentajes en peso de $\mathrm{Si}+\mathrm{Al}+\mathrm{Fe}$ mayores al $65 \%$ y de $\mathrm{Ca}$ menores al $10 \%$ (ver Tabla 1).

Dicha afirmación es confirmada por el análisis estructural y de fases usando difracción de rayos $\mathrm{X}$, el cual se presenta a continuación.

El patrón de difracción de rayos $\mathrm{X}(\mathrm{DRX})$ para la muestra en polvo de ceniza volante de Termotasajero, se presenta en la Figura 10. En este se aprecia la presencia de fase cristalina y de fase vítrea o amorfa, esta última se observa en la discontinuidad lineal y en la curvatura de la base del patrón de difracción.

El análisis cualitativo de fases hecho al patrón de DRX, reveló la presencia de las fases mineralógicas: Cuarzo, Mullite, Hematite y Brucite.

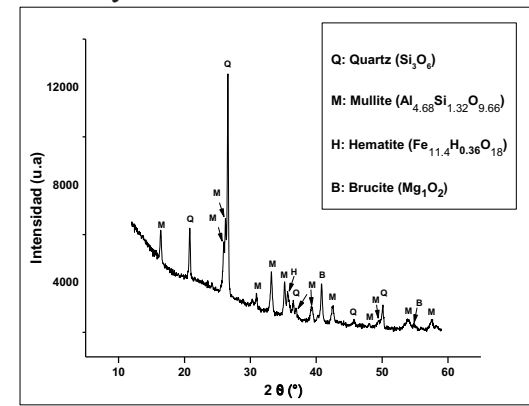

Figura 10. Patrón de difracción de rayos X (DRX) de la muestra de ceniza volante suministrada por la empresa Termotasajero S.A.

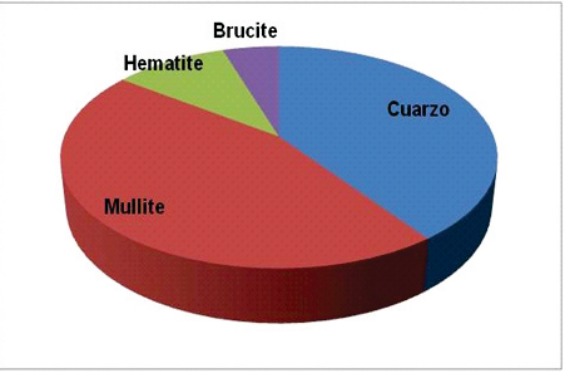

Figura 11. Porcentaje en peso (\%wt) de las fases mineralógicas presentes en la muestra de cenizas volantes de Termotasajero S.A.

Al efectuar un análisis semicuantitativo a las fases minerales presentes en el patrón de difracción — proceso en el que se usó el software X'Pert High Score Plus, el cual viene con el equipo de difracción-, se determinó el porcentaje en peso (\%wt) de cada una de las fases mineralógicas halladas (ver Figura 11), y se encontró que la fase mayoritaria (45\%) es la mullita $\left(\mathrm{Al}_{4.68} \mathrm{Si}_{1.32} \mathrm{O}_{9.66}\right)$, seguida del cuarzo $\left(\mathrm{Si}_{3} \mathrm{O}_{6}\right)$ con un 40 $\%$, y de la hematita $\left(\mathrm{Fe}_{11.40} \mathrm{H}_{0.36} \mathrm{O}_{18}\right)$ con un $10 \%$, y en menor concentración, la Brucite u óxido de magnesio hidratado $\left(\mathrm{Mg}_{1} \mathrm{O}_{2}\right)$.

Desde el punto de vista estructural, se encontró que los sistemas cristalinos de cada una de las fases mineralógicas halladas son: 
El cuarzo $\left(\mathrm{Si}_{3} \mathrm{O}_{6}\right)$, el cual se halló usando el patrón de referencia de la ICDD, con código No. 96-900-9667. Dicha estructura corresponde a un sistema cristalino hexagonal, con parámetros de la celda unitaria o red $\mathbf{a}=\mathbf{b}=4.9160 \AA$, $\mathbf{c}=5.4090 \AA, \boldsymbol{\alpha}=\boldsymbol{\beta}=90^{\circ},=120^{\circ}$.

La Mullite $\left(\mathrm{Al}_{4.68} \mathrm{Si}_{1.32} \mathrm{O}_{9.66}\right)$, con una estructura cristalina ortorrómbica, determinada por el código de referencia No. 96-900-5503 de la ICDD, reportando los parámetros de la red cristalina $\mathbf{a}=\mathbf{b}=7.5660 \AA, \mathbf{c}=2.8850 \AA, \boldsymbol{\alpha}=\boldsymbol{\beta}==90^{\circ}$. La presencia de Mullite en la muestra, le confiere estabilidad química, resistencia mecánica, baja dilatación a altas temperaturas, resistencia al choque térmico, a la abrasión mecánica, a la acción corrosiva de gases y al ataque de metales fundidos (Villar, Gago \& García, 2004).

La Hematite $\left(\mathrm{Fe}_{11.40} \mathrm{H}_{0.36} \mathrm{O}_{18}\right)$, correspondiente a un sistema cristalino hexagonal, identificado con el código de referencia No. 96-900-2162 de la ICDD, con parámetros de red $\mathbf{a}=\mathbf{b}=5.0260 \AA, \mathbf{c}=13.7090 \AA, \boldsymbol{\alpha}=\boldsymbol{\beta}=90^{\circ},=120^{\circ}$ Esta fase es la que le confiere el color rojizo a la muestra, y puede ser utilizada en procesos de pulimiento de vidrios y metales preciosos, entre otras aplicaciones.

La Brucite $\left(\mathrm{Mg}_{1} \mathrm{O}_{2}\right)$, hallada según el código de referencia No. 96-900-2355 de la ICDD, correspondiente a un sistema cristalino hexagonal, con parámetros de red $\mathbf{a}=\mathbf{b}=2.9840 \AA$, $\mathbf{c}=4.2940 \AA, \boldsymbol{\alpha}=\boldsymbol{\beta}=90^{\circ},=120^{\circ}$. Se encuentra comúnmente adicionada con la calcita, magnesia, dolomita y con la serpentina principalmente.

Para hallar el porcentaje de cristalinidad o índice de cristalinidad (\%IC) de la muestra, se utilizó el método reportado por Merino y Morales (2008), que consiste en analizar el patrón de difracción para el pico de mayor intensidad, sumando las intensidades de los picos menores y dividida por el valor de la intensidad del mayor pico. En dicho proceso se encontró que el pico más intenso está entre $2 \mathrm{O}=25$ y $27^{\circ}$, y que corresponde a la fase del cuarzo. Los cálculos del \%IC se llevaron a cabo usando el software OriginPro versión 7.0. En la Figura 12 se presenta una amplificación del patrón de difracción correspondiente a la reflexión del pico más intenso de la fase del cuarzo. De éste se encontró que el IC $=14.25 \%$, es decir, que la muestra está formada por un $14.25 \%$ de fase cristalina y un $85.75 \%$ de fase vítrea o amorfa.

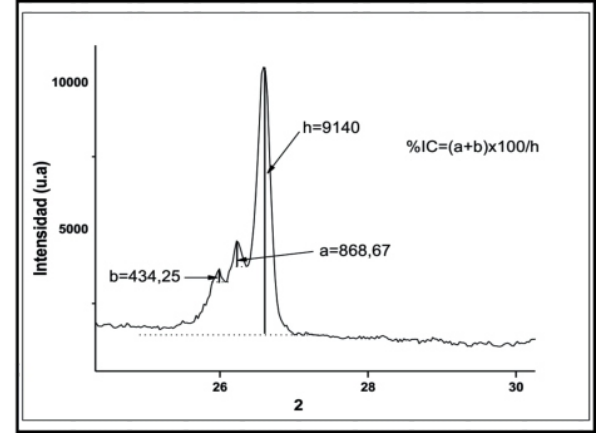

Figura 12. Amplificación del pico de mayor intensidad del patrón de difracción para la muestra de ceniza volante de Termotasajero, usado para hallar el porcentaje IC.
Los resultados hallados usando DRX para las muestras de ceniza volante, son consistentes con los reportados en la literatura para otras cenizas volantes provenientes de centrales térmicas (Cañadas-Serrano et al., 1990; De Luxan, Sánchez \& Soria, 1988; Potgieter, Bada \& PotgieterVermaak, 2009; Iver \& Scott, 2001).

\section{CONCLUSIONES}

Los resultados obtenidos en el análisis granulométrico de las cenizas volantes para la distribución del tamaño de partícula, tienden a situarse en la misma zona. Así mismo se concluye que las cenizas volantes tienen granulometría con tamaño de partícula inferior a $250 \mu \mathrm{m}$, y que del 60 al $90 \%$ son inferiores a $75 \mu \mathrm{m}$; que posee cenosferas de ceniza volante con dimensiones de $6,61,11,59, \mathrm{y} 13,81 \mu \mathrm{m}$ respectivamente y plerosferas que contienen partículas más pequeñas en su interior.

La composición química por elemento usando corrección ZAF a las líneas $\mathrm{K}$ de energía del espectro de dispersión de energías (EDS), se realizó en forma puntual para una partícula de ceniza volante, así como para una zona de inquemados. De lo cual se infiere que las cenizas de Termotasajero son silicoaluminosas con propiedades puzolánicas clase F (según norma ASTM C618), por tener porcentajes en peso de $\mathrm{Si}+\mathrm{Al}+\mathrm{Fe}$ mayores al $65 \%$ y de $\mathrm{MgO}$ menores al $10 \%$.

Las fases inorgánicas con sus estructuras presentes en la ceniza volante, fueron: mullita $\left(\mathrm{Al}_{4.68} \mathrm{Si}_{1.32} \mathrm{O}_{9.66}\right)(45 \%)$, seguido del cuarzo $\left(\mathrm{Si}_{3} \mathrm{O}_{6}\right)$ con un $40 \%$, y de la hematita $\left(\mathrm{Fe}_{11.40} \mathrm{H}_{0.36} \mathrm{O}_{18}\right)$ con un $10 \%$, y en menor concentración la Brucite u óxido de magnesio hidratado $\left(\mathrm{Mg}_{1} \mathrm{O}_{2}\right)$. Así mismo se encontró que la muestra de ceniza volante está conformada por fase cristalinas y amorfa, con un \% IC del $14.25 \%$, y un $85.75 \%$ de fase vítrea.

La composición química y mineralógica de las cenizas volantes de Termotasajero es muy similar a la encontrada para las cenizas volantes de otras centrales termoeléctricas

\section{REFERENCIAS}

Bouzoubaa, N., Zhang, M. H. \& Malhotra, V. M. (2001). Mechanical properties and durability of concrete made with high-volume fly ash blended cements using a coarse fly ash. Cement and Concrete Research, 31(10), 1393-1402.

Cabrera, J.G. \& Cusens, A.R. (1982).The use of PFA in concrete. International symposium; vol.1 and 2, Leeds.

Cañadas-Serrano, L. et al. (1990). Caracterización de las cenizas de cinco centrales térmicas españolas. Medio ambiente-RETEMA, 13-20.

Davidovits, J. (1994). Global Warming Impact on the Cement and Aggregates Industries. World Resource Review, 6(2), 263-278.
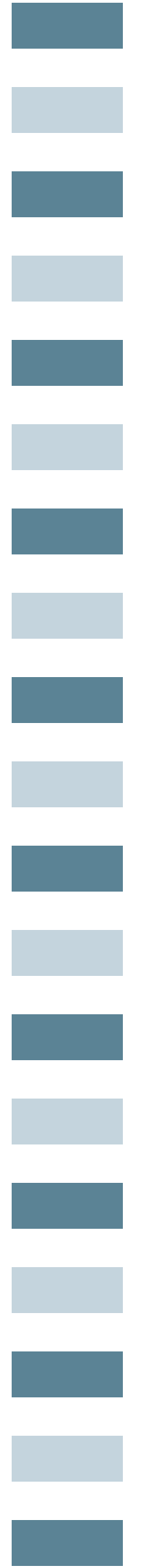
De Luxan, M.P., Sánchez, I. \& Soria, F. (1988). Características de cenizas volantes españolas. Mater. de const. 38 (209), 25-38.

Hanehara, S., Tomosawa, F., Kobayakawa, M. \& Hwang, K. (2001). Effects of water/powder ratio, mixing ratio of fly ash, and curing temperature on pozzolanic reaction of fly ash in cement paste. Cement and Concrete Research, 31(1), 31-39.

Iyer, R.S. \& Scott, J.A. (2001). Power Station Fly Ash- A Review of Value- Added Utilization Outside of the Construction Industry. Resources, Conservation, and Recycling, 31, 217-228.

Merino, L. \& Morales, J. (2008). Relationship of the cristallinity index $(\mathrm{CI})$ with the age and content of $\mathrm{F}$ and $\mathrm{CO}_{3}$ ions in vertébrate fossil samples. Revista Estudios Geológicos, 64(1), 75-87.

Öztürk, N. \& Kavak, D. (2005). Adsorption of boron from aqueous solutions using fly ash: Batch and column studies. Journal of Hazardous Materials. B127, 8188.

Potgieter, J.H., Bada, S.O. \& Potgieter-Vermaak, S.S. (2009). Adsorptive removal of various phenols from water by Sout African coal fly ash. Water, 35(1), 89-96.
Rajamannan, B., Kalyana Sundaram, C., Viruthagiri, G. \& Shanmugam, N. (2013). Effect of fly ash addition on the mechanical and other properties of ceramic tiles. International Journal of Latest Research in Science and Technology. 2(1), 486-491.

Ramírez J. 1. (1990). Orígenes, tipos y caracterización de las cenizas volantes. Madrid: MOPU_CEDEX, Cuadernos de Investigación C27.

Ruiz-Román, J.M., Alonso, C., Cambronero, F. Corpas, L.E.G., Alfonso, M. \& Moraño, A.J. (2000). Aprovechamiento de las cenizas volantes, clase F, de centrales térmicas para la fabricación de materiales cerámicos. Bol. Soc. Esp. Cerám. Vidrio, 39(3), 229-231.

Škvára, F., Jílek, T. \& Kopecký, L. (2005). Geopolymer materials based on fly ash". Ceramics - Silikáty, 49 (3), 195-204.

Villar, M.P., Gago, L. \& García, R. (2004). Comportamiento de Mullitas a alta temperaturas. Estudio mediante XRD. Bol. Soc. Esp. Cerám. V. 43 (2), 135-137.

Wang, S., Boyjoo, Y., Choueib, A. \& Zhu, Z. H. (2005). Removal of dyes from aqueous solution using fly ash and red mud. Water Research, 39(1), 129-138. 